\title{
Sesqui-Harmonic Curves in LP-Sasakian Manifolds
}

\author{
Müslüm Aykut Akgün ${ }^{1 *}$ and Bilal Eftal Acet ${ }^{2}$ \\ ${ }^{1}$ Department of Mathematics, Technical Sciences Vocational High School, Adiyaman University, Adiyaman, Turkey \\ ${ }^{2}$ Department of Mathematics, Faculty of Science and Arts, Adlyaman University, Adiyaman, Turkey \\ * Corresponding author
}

\section{Article Info}

Keywords: Frenet curves, LP-Sasakian manifolds, Sesqui-harmonic Map 2010 AMS: 53C25, 53C42, 53C50.

Received: 17 August 2021

Accepted: 29 September 2021

Available online: 1 October 2021

\begin{abstract}
In this article, we characterize interpolating sesqui-harmonic spacelike curves in a fourdimensional conformally and quasi-conformally flat and conformally symmetric Lorentzian Para-Sasakian manifold. We give some theorems for these curves.
\end{abstract}

\section{Introduction}

Let $\left(M_{1}, g_{1}\right)$ and $\left(M_{2}, g_{2}\right)$ be Riemannian manifolds and $\sigma:\left(M_{1}, g_{1}\right) \rightarrow\left(M_{2}, g_{2}\right)$ be a smooth map. The equation

$$
\mathbb{L}(\sigma)=\frac{1}{2} \int_{M_{1}}|d \sigma|^{2} \vartheta_{g_{1}}
$$

gives the critical points of energy functional The Euler-Lagrange equation of the energy functional gives the harmonic equation defined by vanishing of

$$
\tau(\sigma)=\operatorname{trace} \nabla d \sigma
$$

where $\tau(\sigma)$ is called the tension field of the map $\sigma$.

Biharmonic maps between Riemannian manifolds were studied in [1]. Biharmonic maps between Riemannian manifolds $\psi:\left(M_{1}, g_{1}\right) \rightarrow$ $\left(M_{2}, g_{2}\right)$ are the critical points of the bienergy functional

$$
\mathbb{L}_{2}(\sigma)=\frac{1}{2} \int_{M_{1}}|\tau(\sigma)|^{2} \vartheta_{g_{1}}
$$

In [2], G.Y. Jiang derived the variations of bienergy formulas and showed that

$$
\begin{aligned}
\tau_{2}(\sigma) & =-J^{\sigma}(\tau(\sigma)) \\
& =-\Delta \tau(\Psi)-\operatorname{traceR}^{N}(d \sigma, \tau(\sigma)) d \sigma,
\end{aligned}
$$

where $J^{\sigma}$ is the Jacobi operator of $\sigma$. The equation $\tau_{2}(\sigma)=0$ is called biharmonic equation.

Interpolating sesqui-harmonic maps were studied by Branding [3]. The author defined an action functional for maps between Riemannian manifolds that interpolated between the actions for harmonic and biharmonic maps. $\Psi$ is interpolating sesqui-harmonic if it is critical point of $\delta_{1}, \delta_{2}(\Psi)$,

$$
\mathbb{L}_{\delta_{1}, \delta_{2}}(\Psi)=\delta_{1} \int_{M_{1}}|d \Psi|^{2} v_{g_{1}}+\delta_{2} \int_{M_{1}}|\tau(\Psi)|^{2} v_{g_{1}},
$$

where $\delta_{1}, \delta_{2} \in \mathbb{R}[3]$. 
For $\delta_{1}, \delta_{2} \in \mathbb{R}$ the equation

$$
\tau_{\delta_{1}, \delta_{2}}(\Psi)=\delta_{2} \tau_{2}(\Psi)-\delta_{1} \tau(\Psi)=0,
$$

is the interpolating sesqui-harmonic map equation [3].

An interpolating sesqui-harmonic map is biminimal if variations of (1.1) that are normal to the image $\Psi\left(M_{1}\right) \subset M_{2}$ and $\delta_{2}=1, \delta_{1}>0$ [4] In a 3-dimensional sphere, interpolating sesqui-harmonic curves were studied in [3]. Interpolating sesqui-harmonic Legendre curves in Sasakian space forms were characterized in [5]. Recently, Yüksel Perktaş et all. introduced biharmonic and biminimal Legendre curves in 3-dimensional $f$-Kenmotsu manifold [6]. Moreover, spacelike and timelike curves characterized in a four dimensional manifold to be proper biharmonic in [7]. Motivated by the above studies, in this paper, we examine interpolating sesqui-harmonic curves in 4-dimensional LP-Sasakian manifold.

\section{Preliminaries}

\subsection{Lorentzian almost paracontact manifolds}

Let $M$ be an $n$-dimensional differentiable manifold equipped with a structure $(\phi, \zeta, \eta)$, where $\phi$ is a $(1,1)$-tensor field, $\xi$ is a vector field, $\eta$ is a 1 -form on $M$ such that [8]

$$
\begin{gathered}
\phi^{2}=I d+\eta \otimes \zeta \\
\eta(\zeta)=-1 .
\end{gathered}
$$

Also, we have

$$
\eta \circ \phi=0, \quad \phi \zeta=0, \quad \operatorname{rank}(\phi)=n-1 .
$$

If $M$ admits a Lorentzian metric $g$, such that

$$
g(\phi V, \phi W)=g(V, W)+\eta(V) \eta(W)
$$

then $M$ is said to admit a Lorentzian almost paracontact structure $(\phi, \zeta, \eta, g)$.

The manifold $M$ endowed with a Lorentzian almost paracontact structure $(\phi, \zeta, \eta, g)$ is called a Lorentzian almost paracontact manifold [8,9]. In equations (2.1) and (2.2) if we replace $\zeta$ by $-\zeta$, we obtain an almost paracontact structure on $M$ defined by I. Sato [10].

A Lorentzian almost paracontact manifold $(M, \phi, \zeta, \eta, g)$ is called a Lorentzian para-Sasakian manifold [8] if

$$
\left(\nabla_{V} \phi\right) W=g(V, W) \zeta+\eta(W) V+2 \eta(V) \eta(W) \zeta .
$$

It is well konown that, conformal curvature tensor $\tilde{C}$ is given by

$$
\tilde{C}(V, W) Z=R(V, W) Z-\frac{1}{n-2}\{S(W, Z) V-S(V, Z) W+g(W, Z) V-g(V, Z) Q W\}+\left(\frac{r}{(n-1)(n-2)}\right)\{g(W, Z) V-g(V, Z) W\},
$$

where $S$ is the Ricci tensor and $r$ is the scalar curvature. If $C=0$, then Lorentzian para-Sasakian manifold is called conformally flat. Also, quasi conformal curvature tensor $\hat{C}$ is defined by

$\hat{C}(V, W) Z=\alpha R(V, W) Z-\beta\{S(W, Z) V-S(V, Z) W+g(W, Z) Q V-g(V, Z) Q W\}-\left(\frac{r}{n}\left(\frac{\alpha}{(n-1)}+2 \beta\right)\right)\{g(W, Z) V-g(V, Z) W\}$,

where $\alpha, \beta$ constants such that $\alpha \beta \neq 0$. If $\hat{C}=0$, then Lorentzian para-Sasakian manifold is called quasi conformally flat.

A conformally flat and quasi conformally flat LP-Sasakian manifold $M^{n}(n>3)$ is of constant curvature 1 and also a LP-Sasakian manifold is locally isometric to a Lorentzian unit sphere if the relation $R(V, W) \cdot C=0$ holds [11]. For a conformally symmetric Riemannian manifold [12], we have $\nabla C=0$. So, for a conformally symmetric space $R(V, W) \cdot C=0$ satisfies. Therefore a conformally symmetric LP-Sasakian manifold is locally isometric to a Lorentzian unit sphere [11].

In this case, for conformally flat, quasi conformally flat and conformally symmetric LP-Sasakian manifold $M$, for every $V, W, Z \in T M[11]$, we have

$$
R(V, W) Z=g(W, Z) V-g(V, Z) W
$$

\section{Main results}

In this section, we give our main results about interpolating sesqui-harmonic curves in a conformally flat, quasi conformally flat and conformally symmetric LP-Sasakian manifold $\tilde{M}$. From now on, we will consider such a manifold as $\tilde{M}$.

Theorem 3.1. Let $\tilde{M}$ be a 4-dimensional LP-Sasakian manifold and $\gamma: I \rightarrow \tilde{M}$ be a curve parametrized by arclength $s$ with $\left\{t, n, b_{1}, b_{2}\right\}$ orthonormal Frenet frame such that first binormal vector $b_{1}$ is timelike. Then $\gamma$ is a interpolating sesqui-harmonic curve if and only if either

i) $\gamma$ is a circle with $\rho_{1}=\sqrt{1-\frac{\delta_{1}}{\delta_{2}}}$,
or

ii) $\gamma$ is a helix with $\rho_{1}^{2}-\rho_{2}^{2}=1-\frac{\delta_{1}}{\delta_{2}}$

where $\frac{\delta_{1}}{\delta_{2}}<1$. 
Proof. Let $\tilde{M}$ be a four-dimensional LP-Sasakian manifold and $\gamma$ be a parametrized curve on $\tilde{M}$. If the first binormal vector $b_{1}$ of $\left\{t, n, b_{1}, b_{2}\right\}$ orthonormal Frenet frame is a timelike vector, then the Frenet equations of the curve $\gamma$ given as

$$
\left[\begin{array}{c}
\nabla_{t} t \\
\nabla_{t} n \\
\nabla_{t} b_{1} \\
\nabla_{t} b_{2}
\end{array}\right]=\left[\begin{array}{cccc}
0 & \rho_{1} & 0 & 0 \\
-\rho_{1} & 0 & \rho_{2} & 0 \\
0 & \rho_{2} & 0 & \rho_{3} \\
0 & 0 & \rho_{3} & 0
\end{array}\right]\left[\begin{array}{c}
t \\
n \\
b_{1} \\
b_{2}
\end{array}\right]
$$

where $\rho_{1}, \rho_{2}, \rho_{3}$ are respectively the first, the second and the third curvature of the curve $\gamma$ [13].

By using (3.1) and equation (2.5), we obtain

$$
\begin{gathered}
\nabla_{t} t=\rho_{1} n, \\
\nabla_{t} \nabla_{t} t=-\rho_{1}^{2} t+\rho_{1}^{\prime} n+\rho_{1} \rho_{2} b_{1}, \\
\nabla_{t} \nabla_{t} \nabla_{t} t=-\left(3 \rho_{1} \rho_{1}^{\prime}\right) t+\left(\rho_{1}^{\prime \prime}-\rho_{1}^{3}+\rho_{1} \rho_{2}^{2}\right) n+\left(2 \rho_{1}^{\prime} \rho_{2}+\rho_{1} \rho_{2}^{\prime}\right) b_{1}+\left(\rho_{1} \rho_{2} \rho_{3}\right) b_{2},
\end{gathered}
$$

and

$$
R\left(t, \nabla_{t} t\right) t=-\rho_{1} n
$$

Considering above equations in (1.2), we have

$$
\tau_{\delta_{1}, \delta_{2}}(\Psi)=-\left(3 \rho_{1} \rho_{1}^{\prime}\right) \delta_{2} t+\left\{\begin{array}{c}
\left(\rho_{1}^{\prime \prime}-\rho_{1}^{3}+\rho_{1} \rho_{2}^{2}+\rho_{1}\right) \delta_{2} \\
-\rho_{1} \delta_{1}
\end{array}\right\} n+\left(2 \rho_{1}^{\prime} \rho_{2}+\rho_{1} \rho_{2}^{\prime}\right) \delta_{2} b_{1}+\left(\rho_{1} \rho_{2} \rho_{3}\right) \delta_{2} b_{2} .
$$

Thus, $\gamma$ is a interpolating sesqui-harmonic curve if and only if

$$
\begin{gathered}
\rho_{1}=\text { const } .>0 \quad \rho_{2}=\text { const } . \\
\rho_{1}^{2}-\rho_{2}^{2}=1-\frac{\delta_{1}}{\delta_{2}}, \\
\rho_{2} \rho_{3}=0 .
\end{gathered}
$$

So, we get the proof.

Theorem 3.2. Let $\tilde{M}$ be a 4-dimensional LP-Sasakian manifold and $\gamma: I \rightarrow \tilde{M}$ be a curve parametrized by arclength $s$ with $\left\{t, n, b_{1}, b_{2}\right\}$ orthonormal Frenet frame such that second binormal vector $b_{2}$ is timelike. Then $\gamma$ is a interpolating sesqui-harmonic curve if and only if either

i) $\gamma$ is a circle with $\rho_{1}=\sqrt{1-\frac{\delta_{1}}{\delta_{2}}}$,

ii) $\gamma$ is a helix with $\rho_{1}^{2}+\rho_{2}^{2}=1-\frac{\delta_{1}}{\delta_{2}}$

where $\frac{\delta_{1}}{\delta_{2}}<1$.

Proof. Let $\tilde{M}$ be a four-dimensional LP-Sasakian manifold and $\gamma$ be a parametrized curve on $\tilde{M}$. If the vector $b_{2}$ of $\left\{t, n, b_{1}, b_{2}\right\}$ orthonormal Frenet frame is a timelike vector, then the Frenet equations of the curve $\gamma$ given as

$$
\left[\begin{array}{c}
\nabla_{t} t \\
\nabla_{t} n \\
\nabla_{t} b_{1} \\
\nabla_{t} b_{2}
\end{array}\right]=\left[\begin{array}{cccc}
0 & \rho_{1} & 0 & 0 \\
-\rho_{1} & 0 & \rho_{2} & 0 \\
0 & -\rho_{2} & 0 & \rho_{3} \\
0 & 0 & \rho_{3} & 0
\end{array}\right]\left[\begin{array}{c}
t \\
n \\
b_{1} \\
b_{2}
\end{array}\right]
$$

where $\rho_{1}, \rho_{2}, \rho_{3}$ are respectively the first, the second and the third curvature of the curve [13].

From (3.2) and (2.5), we get

$$
\begin{gathered}
\nabla_{t} t=\rho_{1} n, \\
\nabla_{t} \nabla_{t} t=-\rho_{1}^{2} t+\rho_{1}^{\prime} n+\rho_{1} \rho_{2} b_{1}, \\
\nabla_{t} \nabla_{t} \nabla_{t} t=-\left(3 \rho_{1} \rho_{1}^{\prime}\right) t+\left(\rho_{1}^{\prime \prime}-\rho_{1}^{3}-\rho_{1} \rho_{2}^{2}\right) n+\left(2 \rho_{1}^{\prime} \rho_{2}+\rho_{1} \rho_{2}^{\prime}\right) b_{1}+\left(\rho_{1} \rho_{2} \rho_{3}\right) b_{2},
\end{gathered}
$$

and

$$
R\left(t, \nabla_{t} t\right) t=-\rho_{1} n
$$

Considering above equations in (1.2), we have

$$
\tau_{\delta_{1}, \delta_{2}}(\Psi)=-\left(3 \rho_{1} \rho_{1}^{\prime}\right) \delta_{2} t+\left\{\begin{array}{c}
\left(\rho_{1}^{\prime \prime}-\rho_{1}^{3}-\rho_{1} \rho_{2}^{2}+\rho_{1}\right) \delta_{2} \\
-\rho_{1} \delta_{1}
\end{array}\right\} n+\left(2 \rho_{1}^{\prime} \rho_{2}+\rho_{1} \rho_{2}^{\prime}\right) \delta_{2} b_{1}+\left(\rho_{1} \rho_{2} \rho_{3}\right) \delta_{2} b_{2} .
$$


In this case, $\gamma$ is a interpolating sesqui-harmonic curve if and only if

$$
\begin{gathered}
\rho_{1}=\text { const. }>0 \quad \rho_{2}=\text { const } . \\
\rho_{1}^{2}+\rho_{2}^{2}=1-\frac{\delta_{1}}{\delta_{2}}, \\
\rho_{2} \rho_{3}=0 .
\end{gathered}
$$

This equation proves our assertion.

Theorem 3.3. Let $\tilde{M}$ be a 4-dimensional LP-Sasakian manifold and $\gamma: I \rightarrow \tilde{M}$ be a curve parametrized by arclength $s$ with $\left\{t, n, b_{1}, b_{2}\right\}$ orthonormal Frenet frame such that binormal vector $b_{1}$ is null. Then $\gamma$ is a interpolating sesqui-harmonic curve if and only if either

i) $\rho_{1}=\sqrt{1-\frac{\delta_{1}}{\delta_{2}}}$ and

and

ii) $\rho_{2}=0$ or $|\ln | \rho_{2}(s)=-\int \rho_{3}(s) d s$.

Proof. Let $\tilde{M}$ be a four-dimensional LP-Sasakian manifold and $\gamma$ be a parametrized curve on $\tilde{M}$. If the first binormal vector $b_{1}$ of $\left\{t, n, b_{1}, b_{2}\right\}$ orthonormal Frenet frame is a null(lightlike) vector, then the Frenet equations of the curve $\gamma$ given as

$$
\left[\begin{array}{c}
\nabla_{t} t \\
\nabla_{t} n \\
\nabla_{t} b_{1} \\
\nabla_{t} b_{2}
\end{array}\right]=\left[\begin{array}{cccc}
0 & \rho_{1} & 0 & 0 \\
-\rho_{1} & 0 & \rho_{2} & 0 \\
0 & 0 & \rho_{3} & 0 \\
0 & \rho_{2} & 0 & -\rho_{3}
\end{array}\right]\left[\begin{array}{c}
t \\
n \\
b_{1} \\
b_{2}
\end{array}\right]
$$

where $\rho_{1}, \rho_{2}, \rho_{3}$ are respectively the first, the second and the third curvature of the curve [13].

By use of (3.3) and equation (2.5), we have

$$
\begin{gathered}
\nabla_{t} t=\rho_{1} n, \\
\nabla_{t} \nabla_{t} t=-\rho_{1}^{2} t+\rho_{1}^{\prime} n+\rho_{1} \rho_{2} b_{1}, \\
\nabla_{t} \nabla_{t} \nabla_{t} t=-\left(3 \rho_{1} \rho_{1}^{\prime}\right) t+\left(\rho_{1}^{\prime \prime}-\rho_{1}^{3}+\rho_{1}\right) n+\left(2 \rho_{1}^{\prime} \rho_{2}+\rho_{1} \rho_{2}^{\prime}\right) b_{1}+\left(\rho_{1} \rho_{2} \rho_{3}\right) b_{2},
\end{gathered}
$$

and

$$
R\left(t, \nabla_{t} t\right) t=-\rho_{1} n
$$

In view of (1.2), we arrive at

$$
\tau_{\delta_{1}, \delta_{2}}(\Psi)=-\left(3 \rho_{1} \rho_{1}^{\prime}\right) \delta_{2} t+\left\{\begin{array}{c}
\left(\rho_{1}^{\prime \prime}-\rho_{1}^{3}+\rho_{1}\right) \delta_{2} \\
-\rho_{1} \delta_{1}
\end{array}\right\} n+\left(2 \rho_{1}^{\prime} \rho_{2}+\rho_{1} \rho_{2}^{\prime}\right) \delta_{2} b_{1}+\left(\rho_{1} \rho_{2} \rho_{3}\right) \delta_{2} b_{2} .
$$

Thus, $\gamma$ is a interpolating sesqui-harmonic curve if and only if

$$
\begin{gathered}
\rho_{1} \rho_{1}^{\prime}=0 \\
\left(\rho_{1}^{\prime \prime}-\rho_{1}^{3}+\rho_{1}\right) \delta_{2}-\rho_{1} \delta_{1}=0 \\
2 \rho_{1}^{\prime} \rho_{2}+\rho_{1} \rho_{2}^{\prime}+\rho_{1} \rho_{2} \rho_{3}=0
\end{gathered}
$$

If we consider non-geodesic solution, we obtain

$$
\begin{aligned}
& \rho_{1}=\sqrt{1-\frac{\delta_{1}}{\delta_{2}}}, \\
& \rho_{2}^{\prime}+\rho_{2} \rho_{3}=0,
\end{aligned}
$$

where $\frac{\delta_{1}}{\delta_{2}}<1$.

Theorem 3.4. Let $\tilde{M}$ be a 4-dimensional LP-Sasakian manifold and $\gamma: I \rightarrow \tilde{M}$ be a curve parametrized by arclength $s$ with $\left\{t, n, b_{1}, b_{2}\right\}$ orthonormal Frenet frame such that normal vector $n$ is timelike. Then $\gamma$ is a interpolating sesqui-harmonic curve if and only if either i) $\gamma$ is a circle with $\rho_{1}=\sqrt{\frac{\delta_{1}}{\delta_{2}}-1}$,

or

ii) $\gamma$ is a helix with $\rho_{1}^{2}+\rho_{2}^{2}=\frac{\delta_{1}}{\delta_{2}}-1$ where $\frac{\delta_{1}}{\delta_{2}}>1$. 
Proof. Let $\tilde{M}$ be a four-dimensional LP-Sasakian manifold and $\gamma$ be a parametrized curve on $\tilde{M}$. If the normal vector $n$ of $\left\{t, n, b_{1}, b_{2}\right\}$ orthonormal Frenet frame is a timelike vector, then the Frenet equations of the curve $\gamma$ given as

$$
\left[\begin{array}{c}
\nabla_{t} t \\
\nabla_{t} n \\
\nabla_{t} b_{1} \\
\nabla_{t} b_{2}
\end{array}\right]=\left[\begin{array}{cccc}
0 & \rho_{1} & 0 & 0 \\
\rho_{1} & 0 & \rho_{2} & 0 \\
0 & \rho_{2} & 0 & \rho_{3} \\
0 & 0 & -\rho_{3} & 0
\end{array}\right]\left[\begin{array}{c}
t \\
n \\
b_{1} \\
b_{2}
\end{array}\right]
$$

where $\rho_{1}, \rho_{2}, \rho_{3}$ are respectively the first, the second and the third curvature of the curve [13].

By using (3.4) and equation (2.5), we obtain

$$
\begin{gathered}
\nabla_{t} t=\rho_{1} n \\
\nabla_{t} \nabla_{t} t=-\rho_{1}^{2} t+\rho_{1}^{\prime} n+\rho_{1} \rho_{2} b_{1}, \\
\nabla_{t} \nabla_{t} \nabla_{t}=-\left(3 \rho_{1} \rho_{1}^{\prime}\right) t+\left(\rho_{1}^{\prime \prime}+\rho_{1}^{3}+\rho_{1} \rho_{2}^{2}+\rho_{1}\right) n+\left(2 \rho_{1}^{\prime} \rho_{2}+\rho_{1} \rho_{2}^{\prime}\right) b_{1}+\left(\rho_{1} \rho_{2} \rho_{3}\right) b_{2},
\end{gathered}
$$

and

$$
R\left(t, \nabla_{t} t\right) t=-\rho_{1} n
$$

Considering above equations in (1.2), we have

$$
\tau_{\delta_{1}, \delta_{2}}(\Psi)=-\left(3 \rho_{1} \rho_{1}^{\prime}\right) \delta_{2} t+\left\{\begin{array}{c}
\left(\rho_{1}^{\prime \prime}-\rho_{1}^{3}+\rho_{1} k_{2}^{2}+\rho_{1}\right) \delta_{2} \\
-\rho_{1} \delta_{1}
\end{array}\right\} n+\left(2 \rho_{1}^{\prime} \rho_{2}+\rho_{1} \rho_{2}^{\prime}\right) \delta_{2} b_{1}+\left(\rho_{1} \rho_{2} \rho_{3}\right) \delta_{2} b_{2} .
$$

Thus, $\gamma$ is a interpolating sesqui-harmonic curve if and only if

$$
\begin{gathered}
\rho_{1}=\text { const. }>0 \quad \rho_{2}=\text { const } . \\
\rho_{1}^{2}+\rho_{2}^{2}=\frac{\delta_{1}}{\delta_{2}}-1, \\
\rho_{2} \rho_{3}=0 .
\end{gathered}
$$

So, we get the proof.

\section{Conclusion}

In this paper we charaecterized spacelike curves to be Sesqui-harmonic curves in LP-Sasakian manifolds. We gave four theorems about these curves. These theorems showed that if we change the vector fields of the Frenet frame $\left\{t, n, b_{1}, b_{2}\right\}$, then the equation of Sesqui-harmonic curves change. So, we introduced four different spacelike Sesqui-harmonic curves in this manner.

\section{Acknowledgements}

The authors would like to express their sincere thanks to the editor and the anonymous reviewers for their helpful comments and suggestions.

\section{Funding}

There is no funding for this work.

\section{Availability of data and materials}

Not applicable.

\section{Competing interests}

The authors declare that they have no competing interests.

\section{Author's contributions}

All authors contributed equally to the writing of this paper. All authors read and approved the final manuscript. 


\section{References}

[1] J. Eells, J.H. Sampson, Harmonic mapping of the Riemannian manifold, American J. Math., 86(1964), $109-160$.

[2] G.Y. Jiang, 2-harmonic isometric immersions between Riemannian manifolds, Chinese Ann. Math. Ser. A., 7(1986), $130-144$.

[3] V. Branding, On interpolating sesqui-harmonic maps between Riemannian manifolds, J. Geom. Anal., 30(2020), $278-273$.

[4] L. Loubeau, S. Montaldo, Biminimal immersions, Proc. Edinb. Math. Soc., 51(2008), 421-437.

[5] F. Karaca, C. Özgür, C., De, U. C., On interpolating sesqui-harmonic Legendre curves in Sasakian space forms, Int. J. Geom. Meth. Mod. Phys., 17(1)(2020), 13 pages.

[6] S. Yüksel Perktaş, B.E. Acet, S. Ouakkas, On biharmonic and biminimal curves in 3-dimensional f-Kenmotsu manifolds, Fund. Cont. Math. Sci. 1(1) (2020), 14-22.

[7] S. Keleş, S. Yüksel Perktaş, E. Kılıç,, Biharmonic Curves in LP-Sasakian Manifolds, Bull. Malays. Math. Sci. Soc., 33 (2010), $325-344$.

[8] K. Matsumoto, On Lorentzian paracontact manifolds, Bull. Yamagota Univ. Natur. Sci., 12(1989), 151-156.

[9] K. Matsumoto,I. Mihai, R. Rosca, $\xi$-null geodesic gradient vector fields on a Lorentzian para-Sasakian manifold, J. Korean Math. Soc., 32(1995), 17-31.

[10] I. Sato, On a structure similar to the almost contact structure, Tensor (N.S.)., 30(1976), 219-224.

[11] M. Tarafdar, A. Bhattacharyya, On Lorentzian para-Sasakian manifolds, in Steps in Differential Geometry (Debrecen). Inst. Math. Infor. (2000), 343-348.

[12] M.C. Chaki, B. Gupta, On conformally symmetric spaces, Indian J. Math., 5(1963), 113-122.

[13] J. Walrave, Curves and surfaces in Minkowski spaces, Doctoral Thesis, K. U. Leuven, Fac. of Science, 1995. 